\section{Appearance of Specific Acid Phosphatase Isozymes in the Synovial Fluid of Patients with Rheumatoid Arthritis}

ENZYMEs may occur in multiple molecular forms or isozymes ${ }^{1}$, often showing tissue specific variations ${ }^{1-3}$. In different pathological conditions ${ }^{2}$ and after certain typos of medication ${ }^{4,5}$, alterations in serum isozyme patterns have been observed. These findings suggost that isozymes may be valuable markers in clinical research, for example, for the identification of the tissue origin of enzymes occurring in pathological levels in various body fluids. High levels of acid phosphatase activity have been reported in the synovial fluid ${ }^{6}$ and synovial tissue ${ }^{7}$ of patients with rheumatoid arthritis. This report deals with the appearance of certain acid phosphatase isozymes in the knee-joint synovial fluid of patients with rheumatoid arthritis.

Samples of synovial fluid were obtained by puncture of the knee-joint from thirty-six patients. Out of these thirty-six patients, twenty had the clinical diagnosis rheumatoid arthritis (in most cases confirmed by immunological reactions) and sixteen had other diagnoses such as arthrosis deformans, chronic unspecific synovitis, chondromatosis, osteochondritis and various chronic post-traumatic conditions. Samples of synovial membranes were collected from thirteen patients and serum samples were obtained from nineteen patients.

The synovial membranes were homogenized in a mechanical homogenizer in one volume of physiological saline and afterwards centrifuged. The supernatant fluids and the samples of synovial fluid and serum were examined by means of starch gel electrophoresis using a modification (2/3 dilution) of the discontinuous buffer system by Ashton and Braden ${ }^{8}$. After the electrophoresis the starch gels were preincubated for $30 \mathrm{~min}$ in 0.2 molar acetate buffer $p H 4 \cdot 0$ and afterwards stained for $2 \mathrm{~h}$ in 0.2 molar acetate buffer $p H \quad 5 \cdot 2$ using $\alpha$-naphthyl phosphate as substrate and fast garnet GBC salt as a dye coupler.

In the synovial fluid of seventeen out of the twenty patients with rheumatoid arthritis two distinct acid phosphatase components were found (see Fig. 1). None of the sixteen patients with diagnoses other than rheumatoid arthritis showed this pattern. Four different electrophoretically distinct acid phosphatase components, called $A, B, C$ and $D$ in order of decreasing anodal mobility, have been described in man ${ }^{9}$. The components $A$ and $B$ occurred in the synovial fluid of patients with rheumatoid arthritis. In Fig. 1, a tissue extract of placenta is used to indicate the electrophoretic mobilities of the four components. The $A$ and $B$ components were not found in the sera of the nineteen patients of whom nine had rheumatoid arthritis. A weak acid phosphatase component, with a mobility slightly faster than that of acid phosphatase $D$, is sometimes found in serum and synovial fluid.

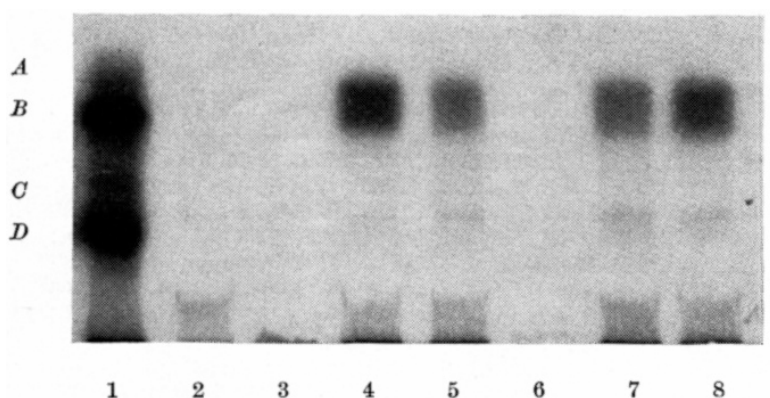

Fig. 1. Photograph of starch gel showing the electrophoretic acid phosphatase patterns in, 1, placental extract and 2-8, synovial fluids Samples $4,5,7$ and 8 are from patients with rheumatoid arthrits, 5 and the direction of migration towards the anode.
In extracts of synovial membranes from thirteen patients there were variations in the acid phosphatase patterns. Six patients with rheumatoid arthritis (and a typical $A B$ acid phosphatase pattern in the synovial fluid) had acid phosphatase components $A, B$ and $D$ in the synovial membrane. Out of seven patients without rheumatoid arthritis, five lacked acid phosphatase activity in the synovial membrane while two had woak acid phosphatases. Treatment with cortisone did not apparently affect the occurrence of acid phosphatase $A$ and $B$ in the synovial fluid. Three patients had an acid phosphatase component in their synovial fluid with a mobility faster than that of the $A$ component. These patients had been given injections of cortisone into the knee-joint.

These preliminary results indicate that the occurrence of acid phosphatase compononts $A$ and $B$ in the synovial fluid is typical of rheumatoid arthritis. The acid phosphatase components are apparently derived from the pathological synovial membrane.

The high frequency of rheumatoid arthritis patients with the "acid phosphatase $A B$ reaction" suggests that this synovial fluid alteration may be developed into a useful clinical test for rheumatoid arthritis.

\section{BECKMAN}

G. BECKMAN

Department of Genetics,

University of Hawaii.

Department of Orthopaedics,

R. LEMPERG*

University of Uppsala.

Received September 4, 1967

${ }^{1}$ Markert, C. L., and Møller, F., Proc. US Nat. Acad. Sci., 45, 753 (1959).

${ }^{2}$ Lawrence, S. H., in The Zymogram in Clinical Medicine, 100 (Thomas, Springfield, 1964).

3 Beckman, I., in Isozyme Variations in Man, 75 (Karger, Basel, 1966).

4 Beckman, L, and Wetterberg, L., Acta Genet., Basel, 17, 314 (1967).

"Arturson, G., Beckman, L., and Persson, B. H., Nature, 214, 1252 (1967).

' Lehman, M. A., Kream, J., and Brogna, D., J. Bone Joint Surg., 46, 1372 (1964).

Luscombe, M., Nature, 197, 1010 (1963).

8 Ashton, G. C., and Braden, A. W. H., A ustral. J. Exp. Biol. Med. Sci, 14, 248 (1969).

Beckman, L., and Beckman, G., Biochem. Genet. (in the press. 1967).

\section{Dissociation of Urease by Glycol and Glycerol}

THE existence of a urease of low molecular weight has previously been noted ${ }^{1-3}$ but in eircumstances that prevented ready characterization. Wo have shown that highly purified preparations of jack bean urease can be reversibly and rapidly dissociated without appreciable change in enzyme activity. The preparative molecular weight of 485,000 (ref. 4 ) is halved in the process.

Urease can be dissolved in 90 per cent propanediol or glycerol in concentrations up to 1 per cent protein. At $p$ H $9 \cdot 2$ (1 part 0.02 molar tris EDTA buffer plus 9 parts diol or glycerol), urease dissociates completely in a few minutes. In the initial experiments, the electrophoresis pattorn produced in acrylamide gel showed only one band, and because the mobility was substantially greater than that of the preparative form, the band observed was termod $\alpha$-urease. Electrophoretic mobilities in gels of varying concentration and the sedimentation velocity in the ultracentrifuge were consistent with a molecular weight about half that of the original protein. It is possible to free the protein of the propanediol if the sample is dialysed against 0.02 molar tris EDTA, $p \mathbf{H} \mathbf{9 \cdot 2}$. Such solutions were used to determine the nolecular weight by the meniscus depletion sedimentation equilibrium method ${ }^{5}$. At concentrations of $1 \mathrm{mg} / \mathrm{ml}$. of protein, the molecular weight is 236,000 , assuming $\bar{v}^{4}=0 \cdot 733$.

When the $p H$ of a solution of $\alpha$-urease is reduced from $9 \cdot 2$ to $7 \cdot 0$, in the absence of propanediol or glycerol, there 